\title{
Differential vegetative and reproductive performances among fifteen guinea grass hybrids ${ }^{(1)}$
}

\author{
José Alfredo Usberti-Filho(2), Roberto Usberti( ${ }^{(3)}$ and Ricardo Stipp Paterniani(4)
}

\begin{abstract}
The main scope of this work was to detect (Panicum maximum Jacq.) genotype differences as to morphoagronomic and seed quality indices, and to establish character correlations useful for determining vegetative and reproductive trends. Besides the flowering cycle, eight phenological and two seed quality traits were scored in a greenhouse randomized complete block experiment, as follows: plant height $(\mathrm{PH})$, reproductive tiller number/overall tiller number (RTN/OTN), panicle number/reproductive tillers (PN/RT), leaf length (LL), leaf width (LW), panicle length (PL), fresh weight (FW), dry weight (DW), number of seeds/g (NS/G) and seed sample physical purity (SPP). Very-early and early-flowering hybrids consistently showed the highest correlation values among flowering cycle and RTN/OTN $\left(\mathrm{r}=-0.59^{* *}\right), \mathrm{PN} / \mathrm{RT}\left(\mathrm{r}=-0.48^{* *}\right), \mathrm{NS} / \mathrm{G}\left(\mathrm{r}=-0.88^{* *}\right)$ and SPP $\left(\mathrm{r}=-0.80^{* *}\right)$ (reproductive parameters) while intermediate and late-flowering hybrids presented the highest values for LL $\left(\mathrm{r}=0.53^{* *}\right), \mathrm{LW}\left(\mathrm{r}=0.60^{* *}\right), \mathrm{PL}\left(\mathrm{r}=0.77^{* *}\right), \mathrm{FW}\left(\mathrm{r}=0.78^{* *}\right)$ and $\mathrm{DW}\left(\mathrm{r}=0.85^{* *}\right)$ (vegetative traits). The implications of these results for plant breeding and forage management purposes are discussed.
\end{abstract}

Index terms: Panicum maximum, flowering, agronomic characters, vegetative propagation.

Diferenças no desempenho vegetativo e reprodutivo entre quinze híbridos de capim-colonião

Resumo - O principal objetivo deste trabalho foi o de determinar as tendências de desempenho vegetativo e reprodutivo de híbridos de capim-colonião (Panicum maximum Jacq.) recém desenvolvidos, com os genótipos variando quanto ao ciclo de florescimento (desde muito precoces até tardios). Além do ciclo do florescimento, oito parâmetros fenológicos e dois de qualidade das sementes foram testados, em experimento em blocos completos ao acaso em casa de vegetação, como segue: altura da planta (AP), número de perfilhos reprodutivos/número total de perfilhos (NPR/NTP), número de panículas/perfilhos reprodutivos (NP/PR), comprimento da folha (CF), largura da folha (LF), comprimento da panícula $(\mathrm{CP})$, peso fresco (PF), peso seco (PS), número de sementes/g $(\mathrm{NS} / \mathrm{G})$ e pureza física das amostras de sementes (PFS). Os híbridos precoces e semiprecoces apresentaram sempre os maiores valores de correlação obtidos entre ciclos de florescimento e NPR/NTP $(r=-0,59 * *)$ NP/PR $\left(r=-0,48^{* *}\right)$, $\mathrm{NS} / \mathrm{G}\left(\mathrm{r}=-0,88^{* *}\right)$ e PFS $(\mathrm{r}=-0,80 * *)$ (parâmetros reprodutivos) enquanto os híbridos intermediários e tardios apresentaram valores de correlação mais elevados de CF $\left(\mathrm{r}=0,53^{* *}\right), \mathrm{LF}\left(\mathrm{r}=0,60^{* *}\right), \mathrm{CP}$ $\left(\mathrm{r}=0,77^{* *}\right), \mathrm{PF}\left(\mathrm{r}=0,78^{* *}\right)$ e PS $\left(\mathrm{r}=0,85^{* *}\right)$ (características vegetativas). Discutem-se as implicações desses resultados para o melhoramento genético e o manejo de pastagens.

Termos para indexação: Panicum maximum, floração, características agronômicas, propagação vegetativa.

(1) Accepted for publication on January 23, 2001.

(2) Instituto Agronômico, Seção de Genética, Caixa Postal 28, Campinas, SP. E-mail: usberti@eec.iac.br

(3) Coordenadoria de Assistência Técnica Integral, Caixa Postal 1291, CEP 13073-001 Campinas, SP. E-mail: usberti@cati.sp.gov.br

(4) Universidade Estadual de Campinas, Fac. de Engenharia Agrícola, Caixa Postal 6011, CEP 13083-970 Campinas, SP.E-mail: epater@merconet.com.br

\section{Introduction}

Guinea grass (Panicum maximum Jacq.) is a warm-season perennial bunchgrass widely grown as a forage crop in tropical and warm-temperate regions of both hemispheres. Like most tropical grasses, it is a facultative apomictic species, where apospory and pseudogamy occur during the reproductive process. 
Due to the important role of guinea grass in the expansion of animal production, a number of research projects have been carried out on this species in recent years, covering a wide range of topics including: plant breeding (Sukhchain \& Sidhu, 1991; Segui \& Machado, 1992; Noirot, 1993; Sukhchain \& Sidhu, 1993); cytology (Nakagawa et al., 1993; Hamoud et al., 1994; Naumova \& Willemse, 1995); herbage yield and/ or chemical composition (Hill et al., 1989; Santana, 1991; Bayorbor et al., 1992; Kawamoto et al., 1992; Segui et al., 1992; Singh et al., 1995); morphology (Costa et al., 1989; Alcantara et al., 1991); trampling resistance (Sun \& Liddle, 1993); adaptation to acid soils (Thomas \& Lapointe, 1989) among others.

Most of the above studies were performed under field conditions, not taking into account flowering cycle differences among cultivars/introductions, so making data comparisons difficult. In addition, none has been set up to evaluate vegetative and reproductive functions among genotypes widely variable for several traits

The main scope of this research has been to detect genotype differences, as to morphoagronomic and seed quality indices and to establish character correlations, useful to determine vegetative and reproductive trends.

\section{Material and Methods}

Fifteen guinea grass hybrids derived from artificial crossings among previously selected highly sexual (female) and apomictic materials (male) were evaluated (Table 1). Seeds of each hybrid were sown in germination boxes, filled up with a mixture of topsoil, sand and organic matter $(3: 1: 1$ by volume). Fertilizer was added to soil according to soil analysis results. After four weeks, 30 individual seedlings per hybrid were transplanted to plastic bags $(10 \times 15 \mathrm{~cm})$, filled up with the same soil mixture and placed in a greenhouse in a randomized complete block design with three replications (ten plants per replication)

During the experiment, all plastic bags were watered daily. At the onset of the flowering period, the number of days to flowering was scored for each individual plant. As the hybrids reached full blooming, the panicles were packed together in cloth bags (in the same treatment) to avoid seed shattering.

At harvest, the seeds were picked up from the bags and eight vegetative traits (plant height, reproductive tiller number/overall tiller number, panicle number/reproductive tiller, leaf length, leaf width, panicle length, whole plant fresh and dry weights) were scored in each treatment. Additionally, two seed quality tests number of seeds/g and seed sample physical purity) were performed, according to International Seed Testing Association (1985) Plant height has been scored from soil surface to the panicle apex

Measurement data (plant height, leaf length, leaf width, panicle length, fresh and dry weight) were used per se while countings (days-to-flowering, panicle number/reproductive tiller and number of seeds/g) and percentage data (reproductive tiller number/overall tiller number and seed sample physical purity) were transformed to $\mathrm{x}^{0.5}$ and arc $\sin (\mathrm{x} / 100)^{0.5}$, respectively, before statistical analysis.

An ANOVA computer program was used to test differences among hybrids and mean comparisons of different characteristics were made using Duncan's multiple range test. Finally, simple correlations were calculated among flowering cycles and all the quantitative traits studied.

\section{Results and Discussion}

The hybrids studied were ranked in four different groups, according to their flowering cycle: late, intermediate, early and very early-flowering types, using statistical analysis and/or consideration of

Table 1. Pedigree of the 15 guinea grass (Panicum maximum Jacq.) $\mathrm{F}_{1}$ apomictic hybrids ${ }^{(1)}$.

\begin{tabular}{ccl}
\hline Hybrid & Female & \multicolumn{1}{c}{ Male } \\
\hline $\mathrm{H}-10$ & $\mathrm{~T}_{1} \mathrm{SL}$ & SEA-2 \\
$\mathrm{H}-12$ & $\mathrm{~T}_{1} \mathrm{SL}$ & SEA-2 \\
$\mathrm{H}-13$ & $\mathrm{~T}_{1} \mathrm{SL}$ & cv. Tobiatã \\
$\mathrm{H}-21$ & $\mathrm{~T}_{2} \mathrm{SL}$ & PI-277915 \\
$\mathrm{H}-22$ & $\mathrm{~T}_{2} \mathrm{SL}$ & Hybrid-30 \\
$\mathrm{H}-31$ & $\mathrm{~T}_{2} \mathrm{SL}$ & Guineagrass "Dr. Schanks" \\
$\mathrm{H}-33$ & $\mathrm{~T}_{2} \mathrm{SL}$ & Guineagrass "Dr. Schanks" \\
$\mathrm{H}-38$ & $\mathrm{~T}_{2} \mathrm{SL}$ & cv. Aruana \\
$\mathrm{H}-42$ & $\mathrm{~T}_{2} \mathrm{SL}$ & cv. Aruana \\
$\mathrm{H}-54$ & $\mathrm{~T}_{2} \mathrm{SL}$ & cv. Centauro \\
$\mathrm{H}-55$ & $\mathrm{~T}_{2} \mathrm{SL}$ & K-68 \\
$\mathrm{H}-56$ & $\mathrm{~T}_{2} \mathrm{SL}$ & K-68 \\
$\mathrm{H}-64$ & $\mathrm{C}_{1} \mathrm{SL}$ & Hybrid-30 \\
$\mathrm{H}-79$ & $\mathrm{C}_{2} \mathrm{SL}$ & K-68 \\
$\mathrm{H}-140$ & $\mathrm{C}_{7} \mathrm{SL}$ & cv. Aruana \\
\hline
\end{tabular}

${ }^{(1)}$ SEA: South East African; PI: Plant Introduction; T 1 SL: Tobiatã-1 sexual line; $\mathrm{T}_{2} \mathrm{SL}$ : Tobiatã-2 sexual line; $\mathrm{C}_{1} \mathrm{SL}$ : Centauro-1 sexual line; $\mathrm{C}_{2} \mathrm{SL}$ : Centauro-2 sexual line; $\mathrm{C}_{7} \mathrm{SL}$ : Centauro-7 sexual line. 
other forage traits. Five of them $(\mathrm{H}-12, \mathrm{H}-21, \mathrm{H}-32$, $\mathrm{H}-54$ and $\mathrm{H}-64$ ) were classified as late-flowering, five as intermediate-flowering ( $\mathrm{H}-10, \mathrm{H}-13, \mathrm{H}-56, \mathrm{H}-79$ and $\mathrm{H}-55)$, three as early-flowering (H-140, H-22 and $\mathrm{H}-42$ ) and two (H-31 and $\mathrm{H}-33)$ as very early-flowering types (Table 2).

Plant height varied from $2.5 \mathrm{~m}$ (H-56) to $3.4 \mathrm{~m}$ $(\mathrm{H}-54)$; reproductive tiller number/overall tiller number, from $26.3 \%(\mathrm{H}-38)$ to $86.0 \%(\mathrm{H}-22)$; panicle number/reproductive tiller, from 1.2 (H-64, H-56, H-79, $\mathrm{H}-55)$ to 3.4 (H-22); leaf length, from $34.7 \mathrm{~cm}$ (H-140) to $90.9 \mathrm{~cm}$ (H-64); leaf width, from $1.8 \mathrm{~cm}(\mathrm{H}-22$, $\mathrm{H}-140, \mathrm{H}-31, \mathrm{H}-33)$ to $3.5 \mathrm{~cm}$ (H-13); panicle length, from $20.1 \mathrm{~cm}(\mathrm{H}-140)$ to $55.2 \mathrm{~cm}$ (H-64); fresh weight, from $392.4 \mathrm{~g}(\mathrm{H}-140)$ to $1,184.6 \mathrm{~g}(\mathrm{H}-64)$; dry weight, from $116.0 \mathrm{~g}(\mathrm{H}-140)$ to $460.6 \mathrm{~g}(\mathrm{H}-64)$. Seed quality indices scored were number of seeds/g, from 705 $(\mathrm{H}-21)$ to $1,288(\mathrm{H}-31)$ and seed sample physical purity, from $4.8 \%(\mathrm{H}-54)$ to $87.2 \%(\mathrm{H}-31)$.

The genetic materials used revealed wide genetic diversity for all the parameters studied under controlled greenhouse conditions, similar to that observed among cultivars/ecotypes of the same species in field trials (Costa et al., 1989; Alcantara et al., 1991; Segui et al., 1992; Sun \& Liddle, 1993).

Significant high and positive simple correlations were obtained among flowering cycle and plant height $\left(r=0.524^{* *}\right)$; leaf length $\left(r=0.532^{* *}\right)$; leaf width $(r=0.609 * *)$; panicle length $(r=0.775 * *)$; fresh weight $\left(\mathrm{r}=0.788^{* *}\right)$ and dry weight $\left(\mathrm{r}=0.857^{* *}\right)$ (Table 3). Late and intermediate-flowering hybrids showed higher values for vegetative traits. On the other hand, highly significant negative correlations were observed among flowering cycle and reproductive tiller number/overall tiller number $\left(\mathrm{r}=-0.590^{* *}\right)$; panicle number/reproductive tiller $\left(\mathrm{r}=-0.484^{* *}\right)$; number of seeds $/ \mathrm{g}\left(\mathrm{r}=-0.881^{* *}\right)$ and seed sample physical purity $\left(\mathrm{r}=-0.807^{* *}\right)$. As a result, early and very early-flowering hybrids revealed a quite different performance.

Based on these results, the best strategy to preserve the variability within the species should take into consideration the flowering cycle of the available genetic materials. So, a rational and comprehensive germplasm bank should present several groups of ecotypes, introductions and cultivars with different

Table 2. Phenological and seed quality indices recorded on 15 guinea grass (Panicum maximum Jacq.) hybrids in a randomized complete block experiment ${ }^{(1)}$

\begin{tabular}{|c|c|c|c|c|c|c|c|c|c|c|c|c|}
\hline Hybrid & $\begin{array}{c}\text { FC } \\
\text { Days }\end{array}$ & Ranking & $\begin{array}{l}\mathrm{PH} \\
(\mathrm{m})\end{array}$ & $\begin{array}{c}\text { RTN/OTN } \\
(\%)\end{array}$ & $\mathrm{PN} / \mathrm{RT}$ & $\begin{array}{c}\mathrm{LL} \\
(\mathrm{cm})\end{array}$ & $\begin{array}{l}\mathrm{LW} \\
(\mathrm{cm})\end{array}$ & $\begin{array}{r}\mathrm{PL} \\
(\mathrm{cm})\end{array}$ & $\begin{array}{l}\text { FW } \\
(\mathrm{g})\end{array}$ & $\begin{array}{c}\text { DW } \\
(\mathrm{g})\end{array}$ & $\mathrm{NS} / \mathrm{G}$ & $\begin{array}{r}\text { SPP } \\
(\%)\end{array}$ \\
\hline H-12 & $215.7 \mathrm{a}$ & $\mathrm{L}$ & $3.1 \mathrm{bc}$ & $47.7 \mathrm{efg}$ & $1.9 \mathrm{cde}$ & $75.8 \mathrm{~cd}$ & $3.3 \mathrm{ab}$ & $30.6 \mathrm{~cd}$ & $793.0 \mathrm{~b}$ & $341.7 b$ & $715 \mathrm{~h}$ & $16.9 \mathrm{de}$ \\
\hline H-21 & $214.8 \mathrm{a}$ & $\mathrm{L}$ & $3.1 \mathrm{~b}$ & $36.4 \mathrm{gh}$ & $1.5 \mathrm{ef}$ & $74.8 \mathrm{~d}$ & $3.4 \mathrm{ab}$ & $36.1 b c$ & $838.8 b$ & $347.7 \mathrm{~b}$ & $705 \mathrm{~h}$ & 16.1def \\
\hline H-38 & $214.3 \mathrm{a}$ & $\mathrm{L}$ & $3.0 \mathrm{bcd}$ & $26.3 \mathrm{~h}$ & $1.5 \mathrm{def}$ & $74.0 \mathrm{~d}$ & $3.3 b$ & 27.6de & $716.5 b c$ & $290.8 b c$ & $779 \mathrm{~g}$ & 12.0ef \\
\hline H-54 & $205.4 \mathrm{ab}$ & $\mathrm{L}$ & $3.4 \mathrm{a}$ & $58.7 \mathrm{cde}$ & $1.3 \mathrm{f}$ & $90.4 \mathrm{a}$ & $2.8 \mathrm{c}$ & $49.9 \mathrm{a}$ & $933.1 b$ & $343.0 \mathrm{~b}$ & $862 \mathrm{ef}$ & $4.8 \mathrm{f}$ \\
\hline H-64 & $198.2 b$ & $\mathrm{~L}$ & $3.3 \mathrm{a}$ & $58.1 \mathrm{cdef}$ & $1.2 \mathrm{f}$ & $90.9 \mathrm{a}$ & $2.7 \mathrm{~cd}$ & $55.2 \mathrm{a}$ & $1,184.6 \mathrm{a}$ & $460.6 \mathrm{a}$ & $838 f$ & $6.8 \mathrm{ef}$ \\
\hline $\mathrm{H}-10$ & $169.3 \mathrm{c}$ & I & $3.1 \mathrm{~b}$ & 44.2efg & $1.4 \mathrm{f}$ & $81.9 b c$ & $2.3 \mathrm{ef}$ & $40.7 \mathrm{~b}$ & $820.0 \mathrm{~b}$ & $335.3 b$ & $857 \mathrm{ef}$ & $18.1 \mathrm{de}$ \\
\hline $\mathrm{H}-13$ & $139.4 d$ & I & $2.8 \mathrm{de}$ & $67.5 b c$ & $1.9 \mathrm{~cd}$ & $66.4 \mathrm{e}$ & $3.5 \mathrm{a}$ & $41.5 b$ & $551.1 \mathrm{~cd}$ & $233.5 \mathrm{~cd}$ & $685 \mathrm{~h}$ & $30.0 \mathrm{~cd}$ \\
\hline H-56 & $137.7 \mathrm{de}$ & I & $2.5 \mathrm{f}$ & $44.3 \mathrm{efg}$ & $1.2 \mathrm{f}$ & $88.2 \mathrm{ab}$ & $2.4 \mathrm{ef}$ & $48.9 \mathrm{a}$ & $541.2 \mathrm{~cd}$ & $218.2 \mathrm{cde}$ & $948 d$ & $36.9 \mathrm{bc}$ \\
\hline H-79 & 132.1ef & I & $2.5 \mathrm{f}$ & $43.3 \mathrm{fg}$ & $1.2 \mathrm{f}$ & $90.6 \mathrm{a}$ & $2.2 \mathrm{f}$ & $51.1 \mathrm{a}$ & $786.1 b$ & $278.7 \mathrm{bcd}$ & $918 d$ & $44.5 b c$ \\
\hline H-55 & $127.4 \mathrm{fg}$ & I & $2.6 f$ & 51.3defg & $1.2 \mathrm{f}$ & $90.4 \mathrm{a}$ & $2.5 \mathrm{de}$ & $48.8 \mathrm{a}$ & $702.0 \mathrm{bc}$ & 198.8def & $911 \mathrm{de}$ & $41.4 b c$ \\
\hline H-140 & $123.4 \mathrm{gh}$ & $\mathrm{E}$ & $2.6 f$ & 77.7ab & $2.2 \mathrm{bc}$ & $34.7 f$ & $1.8 \mathrm{~g}$ & $20.1 \mathrm{f}$ & $392.4 d$ & 116.0f & $1,044 \mathrm{c}$ & $50.4 b$ \\
\hline H-22 & 118.4hi & $\mathrm{E}$ & $2.9 \mathrm{cde}$ & $86.0 \mathrm{a}$ & $3.4 \mathrm{a}$ & $35.3 \mathrm{f}$ & $1.8 \mathrm{~g}$ & 24.6def & $449.5 d$ & 127.6ef & $1,006 \mathrm{c}$ & $54.6 \mathrm{~b}$ \\
\hline H-42 & $115.2 \mathrm{i}$ & $\mathrm{E}$ & $2.7 \mathrm{ef}$ & $83.7 \mathrm{a}$ & $2.5 b$ & $39.0 \mathrm{f}$ & $1.9 \mathrm{~g}$ & $23.1 \mathrm{ef}$ & $401.0 \mathrm{~d}$ & 138.0ef & $1,133 b$ & $74.7 \mathrm{a}$ \\
\hline H-31 & $91.2 \mathrm{j}$ & VE & $3.0 \mathrm{bcd}$ & $69.2 b c$ & $2.2 b c$ & $38.9 \mathrm{f}$ & $1.8 \mathrm{~g}$ & $23.8 \mathrm{ef}$ & $404.7 d$ & $136.7 \mathrm{ef}$ & $1,288 \mathrm{a}$ & $87.2 \mathrm{a}$ \\
\hline H-33 & $89.8 \mathrm{j}$ & VE & $3.0 \mathrm{bc}$ & $65.4 \mathrm{bcd}$ & $2.3 \mathrm{~b}$ & $40.7 \mathrm{f}$ & $1.8 \mathrm{~g}$ & $24.0 \mathrm{ef}$ & $410.0 \mathrm{~d}$ & 142.0ef & $1,268 \mathrm{a}$ & $85.6 \mathrm{a}$ \\
\hline Mean & 152.9 & & 2.9 & 57.3 & 1.8 & 67.5 & 2.5 & 36.4 & 661.6 & 247.2 & 930.5 & 37.5 \\
\hline C.V.(\%) & 3.1 & & 4.0 & 8.5 & 12.6 & 5.9 & 5.1 & 9.7 & 18.6 & 19.8 & 1.7 & 16.3 \\
\hline
\end{tabular}

(1)Means, in the same column, followed by different letters are statistically different, according to Duncan's multiple range test, at $\mathrm{p}<0.05$; CV: coefficient of variation; FC: flowering cycle (L: late; I: intermediate; E: early and VE: very early); PH: plant height; RTN/OTN: reproductive tiller number/overall tiller number; PN/RT: panicle number/reproductive tiller; LL: leaf length; LW: leaf width; PL: panicle length; FW: fresh weight; DW: dry weight; NS/G: number of seeds/g; SPP: seed sample physical purity 
Table 3. Simple correlation coefficients calculated among flowering cycle and eight phenological/two seed quality indices on 15 guinea grass (Panicum maximum Jacq.) hybrids ${ }^{(1)}$.

\begin{tabular}{|c|c|c|c|c|c|c|c|c|c|c|}
\hline Variable & $\begin{array}{l}\mathrm{PH} \\
(\mathrm{m})\end{array}$ & $\begin{array}{c}\text { RTN/OTN } \\
(\%)\end{array}$ & PN/RT & $\begin{array}{l}\mathrm{LL} \\
(\mathrm{cm})\end{array}$ & $\begin{array}{l}\mathrm{LW} \\
(\mathrm{cm})\end{array}$ & $\begin{array}{l}\text { PL } \\
(\mathrm{cm})\end{array}$ & $\begin{array}{l}\text { FW } \\
(\mathrm{g})\end{array}$ & $\begin{array}{l}\text { DW } \\
(\mathrm{g})\end{array}$ & $\mathrm{NS} / \mathrm{G}$ & $\begin{array}{l}\text { SPP } \\
(\%)\end{array}$ \\
\hline . & $524 * *$ & $-0.590 * *$ & $-0.484 * *$ & $0.532 * *$ & $0.609 * *$ & $0.775^{* *}$ & $0.788 * *$ & $0.857 * *$ & $-0.881 * *$ & $-0.807 * *$ \\
\hline t value & 4.0 & 4.8 & 3.6 & 4.1 & 5.0 & 8.1 & 4.6 & 6.0 & 12.2 & 9.0 \\
\hline
\end{tabular}

${ }^{(1)}$ FC: flowering cycle; PH: plant height; RTN/OTN: reproductive tiller number/overall tiller number; PN/RT: panicle number/reproductive tiller; LL: leaf length; LW: leaf width; PL: panicle length; FW: fresh weight; DW: dry weight; NS/G: number of seeds/g; SPP: seed sample physical purity. **Student $\mathrm{t}$ test significant at $\mathrm{p}<0.01$.

flowering cycles. As a consequence, its maintenance and even the choice of genetic materials for breeding purposes would be easier. In this case, early stageselection schemes would be feasible (for example, when the selection is aimed at short and narrow leaves, in a wide array of genotypes, the selected types, in most cases, will belong to the early or very early-flowering groups).

For forage management purposes, higher pasture persistence should be achieved by using early and very early-flowering hybrids, taking into account their higher seed yielding potentials (high reseeding rates in the field); however, due to their limited forage yields, lower stocking rates should be applied, as compared to those of intermediate and late-flowering cycle genotypes.

\section{Conclusions}

1. The genotypes used reveal wide diversity for all the parameters studied.

2. Significant high and positive simple correlations are obtained among flowering cycle and plant height, leaf length, leaf width, panicle length, fresh weight and dry weight.

3. Late and intermediate-flowering hybrids show higher values for vegetative traits; on the other hand, early and very early-flowering hybrids reveal a significant negative correlation with reproductive parameters.

\section{References}

ALCANTARA, V. P. G.; ALMEIDA, A. R. P.; GHISI, O. M. A. A. Algumas medidas morfofisiológicas em seis cultivares de Panicum maximum Jacq. Revista de Agricultura, Piracicaba, v. 66, p. 47-63, 1991
BAYORBOR, T. B.; KUMAI, S.; FUKUMI, R.; HATTORI, J. Herbage yield, chemical composition and in vitro dry matter digestibility of panic grasses, under 3-cut harvest system and during regrowth period. Journal of the Japanese Society of Grassland Science, TochigiKen, v. 38, p. 315-326, 1992

COSTA, J. C. G.; SAVIDAN, Y. H.; JANK, L.; CASTRO, L. H. R. Morphological studies as a tool for the evaluation of wide tropical forage grass germplasms. In: INTERNATIONAL GRASSLAND CONGRESS, 16. 1989, Nice. Proceedings... Nice: International Grassland Society, 1989. p. 277-278.

HAMOUD, M. A.; HAROUN, A. S.; MACLEOD, R. D.; RICHARDS, A. J. Cytological relationships of selected species of Panicum L. Biologia Plantarum, Dordrecht, v. 36, p. $37-45,1994$.

HILL, K.; WILSON, J. R.; SHELTON, H. M. Yield, persistence and dry matter digestibility of some $\mathrm{C} 3, \mathrm{C} 4$ and C3/C4 Panicum species. Tropical Grasslands, Brisbane, v. 23, p. 240-249, 1989.

INTERNATIONAL SEED TESTING ASSOCIATION (Zurich, Switzerland). International rules for seed testing: rules 1985. Seed Science and Technology, Zürich, v. 13, p. 299-355; 356-513, 1985.

KAWAMOTO, Y.; KINJYO, T.; IKEDA, M.; MIYAGI, E.; HONGO, F.; KOJA, Z. Clipping effects on seasonal dry matter production and nutritive values of tall type grass species of tropical forage. Journal of the Japanese Society of Grassland Science, Tochigi-Ken, v. 38, p. 141-151, 1992.

NAKAGAWA, H.; SHIMIZU, N.; HANNA, W. W. Cytology of "Natsukaze" guinea grass, a natural apomictic hybrid between a sexual and an apomictic plant. Journal of the Japanese Society of Grassland Science, TochigiKen, v. 39, p. 374-380, 1993. 
NAUMOVA, T. N.; WILlEMSE, M. T. M. Ultrastructural characterization of apospory in Panicum maximum. Sexual Plant Reproduction, Berlin, v. 8 p. 197-204, 1995.

NOIROT, M. Allelic ratios and sterility in the agamic complex of the Maximae (Panicoideae): evolutionary role of the residual sexuality. Journal of Evolutionary Biology, Oxford, v. 6, p. 95-101, 1993.

SANTANA, R. R. Seasonal production of 11 Panicum maximum cultivars harvested at a 45-day interval. Journal of Agriculture of the University of Puerto Rico, Rio Piedras, v. 75, p. 61-66, 1991.

SEGUI, E.; MACHADO, H. Estimación de la herdabilidad en hierba de guinea (Panicum maximum Jacq.). Pastos y Forrajes, Havana, v. 15, p. 191-196, 1992.

SEGUI, E.; MACHADO, H.; BLANCO, F. Selección de híbridos en Panicum maximum Jacq. superiores en términos de MS y calidad a los progenitores. Pastos y Forrajes, Havana, v. 15, p. 103-108, 1992
SINGH, D. K.; SINGH, V.; SALE, P. W. G. Effect of cutting management on yield and quality of different selection of guinea grass (Panicum maximum Jacq.) in a humid subtropical environment. Tropical Agriculture, St. Augustine, v. 72, p. 181-187, 1995.

SUKHCHAIN, B. S. S.; SIDHU, B. S. Combining ability analysis for reproductive traits in guinea grass. Tropical Agriculture, St. Augustine, v. 70, p. 252-255, 1993.

SUKHCHAIN, B. S. S.; SIDHU, B. S. Combining ability studies in guinea grass. Crop Improvement, Ludhiana, v. 18 , p. $23-26,1991$.

SUN, D.; LIDDLE, M. J. Plant morphological characteristics and resistance to simulated trampling. Environmental Management, New York, v. 17, p. 511-521, 1993.

THOMAS, D.; LAPOINTE, S. Testing new accessions of guinea grass (Panicum maximum) for acid soils and resistance to spittlebug (Aeneolamia reducta). Tropical Grasslands, Brisbane, v. 23, p. 232-239, 1989. 